\title{
Scanning-less wide-field single-photon counting device for fluorescence intensity, lifetime and time-resolved anisotropy imaging microscopy
}

\author{
J.-A. SPITZ*†\#, R. YASUKUNI*, N. SANDEAU $\ddagger, M$. \\ TAKANO*, J.-J. VACHON*, R. MEALLET-RENAULT* \& R.B. \\ PANSU* \\ *PPSM, Institut d'Alembert, ENS Cachan, CNRS, UniverSud, 61 av President Wilson, \\ F-94230 CACHAN, France \\ $\nmid L B P A$, Institut d'Alembert, ENS Cachan, CNRS, UniverSud, 61 av President Wilson, \\ F-94230 CACHAN, France \\ $\ddagger L P Q M$, Institut d'Alembert, ENS Cachan, CNRS, UniverSud, 61 av President Wilson, \\ F-94230 CACHAN, France \\ \# Present address: MNPS, KULeuven Celestijnenlaan 200F, B-3001 Herverlee, Belgium
}

Key words. Anisotropy, FLIM, fluorescence, Quadrant anode, single-photon counting, tr-FAIM, wide-field imaging.

\section{Summary}

A scanning-less single-photon counting system for FLIM and fluorescence anisotropy wide-field imaging is described and characterized in this paper. The two polarizations of the fluorescence are divided by a Glan prism and acquired at the same time by the $Q_{A}$ detector. Fluorescence decay profiles can be reconstructed for any desired area up to each pixel and used to calculate time-resolved fluorescence anisotropy decays.

\section{Introduction}

Biological and physical sciences have an increasing interest in fluorescence spectroscopy imaging. Fluorescence emission can give access to different parameters such as intensity but also lifetime and anisotropy (Lakowicz, 1999; Valeur, 2001). Indeed, the spectroscopy of fluorescence is a powerful tool to probe viscosity, structural change, functional information and more generally speaking to probe local environment (Fleming, 1986).

Ion indicators where the fluorescence lifetime depends on the chelation state have been found and are used to measure calcium concentration (Schoutteten et al., 1999). FLIM is also used to measure protein contact and deformation through FRET (Förster, 1959). Indeed, the presence of an absorbing dye within a few nanometres of the fluorescent protein induces a reduction of the fluorescence lifetime (Millar, 1996). One

Correspondence to: Dr. Robert B. Pansu. Tel: + 331474027 19; fax: + 3314740 24 54; e-mail:pansu@ppsm.ens-cachan.fr early application of the principle has been in Cameleon, a construction that uses intra-molecular FRET to measure intracellular calcium concentration (Miyawaki et al., 1997).

Anisotropy is a parameter that is used for more general purposes. It measures the randomization of direction of the transition dipole of the emitter. Most of the time this randomization is due to the rotational dynamics of the fluorescent label; however, it can also be due to the energy transfer in a FRET process (Tramier et al., 2000). Quite often a change in the movement of the fluorescent label can be predicted or expected when a biological event occurs and it can be used to measure protein dimerization through FRET between identical labels (Homo FRET) (Gautier et al., 2001).

There are three main FLIM techniques: phase fluorimetry, time-gated set-ups and single-photon counting systems (SPCS) (Herman et al., 1999). The first two are widely used due to the development of CCD cameras (Wang et al., 1992; Gadella etal., 1993; So et al., 1995; So et al., 1998). Although, the latter remains more unconventional, it seems more respectful to the sample because of its increased sensitivity. In the most common SPCS FLIM set-ups (Buurman et al., 1992) that are now commercially available, the laser scans the image pixel by pixel, which slows down the acquisition process. The use of widefield imaging detectors for full parallel pixel acquisition has not been extensively developed (Emiliani et al., 2003). Moreover, instead of sampling the detection, which significantly reduces the sensitivity, each emitted photon is taken into account.

Furthermore, time-resolved fluorescence anisotropy imaging has been described. Jovin et al. developed a technology 


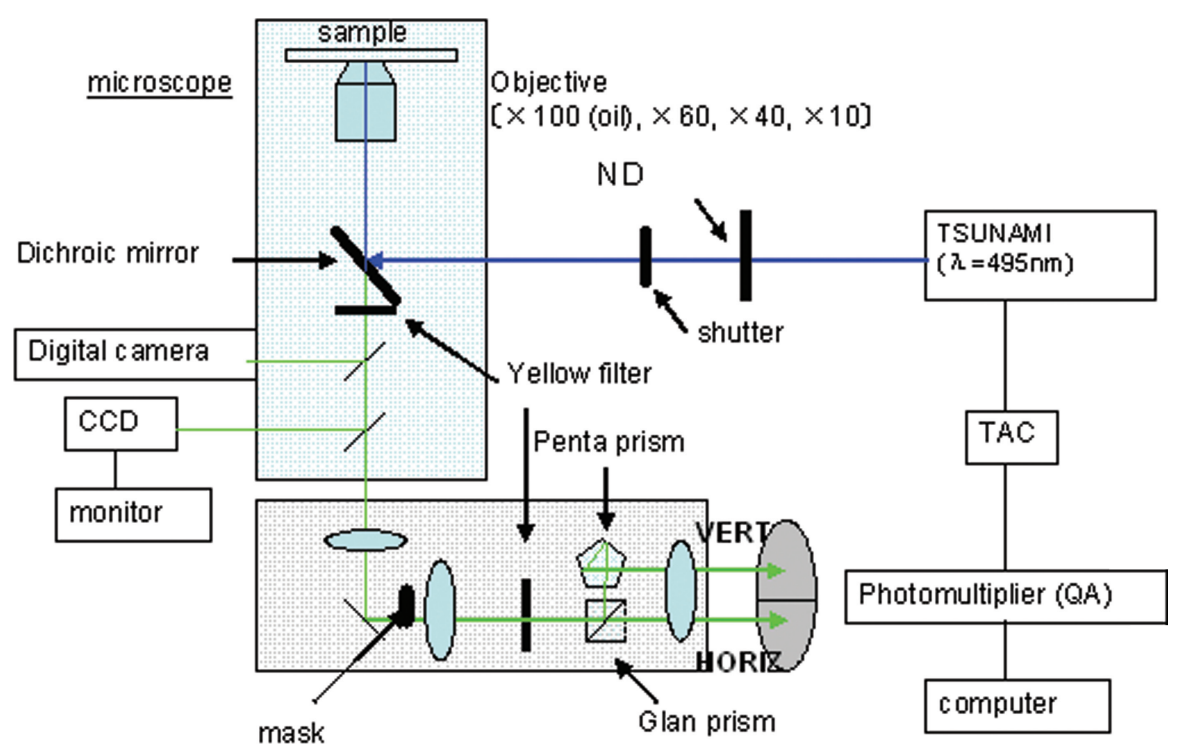

Fig. 1. Experimental set-up.

in which the phase delay and intensity ratios between the polarized components of the fluorescence signal are recorded, leading to estimations of rotational correlation times and limiting anisotropies. This set-up was successfully applied to rotational dynamics studies in solution and emFRET in bacteria expressing EGFP (Clayton et al., 2002). However, in their experiments, the two different polarizations are acquired successively. French et al. are able to image the rotational motility of a fluorophore thanks to a time-gated fluorescence lifetime imaging system (Siegel et al., 2003). Time-resolved fluorescence anisotropy has also been successfully applied to single-molecule spectroscopy (Ha et al., 1999; Schaffer et al., 1999).

In our set-up, the whole decay profile of the two polarizations is acquired simultaneously with a single-photon counting system. Thus, there is no sampling of the fluorescence decay. Moreover, the two polarizations are acquired at the same time, with the same sample, at the same position, with the same experimental conditions preventing us from artefacts such as instrument drift, evolution of the sample or photobleaching.

\section{Materials and methods}

The experimental device is shown in Fig. 1. The excitation source is an argon-ion laser pumped mode-locked Ti/sapphire laser (Tsunami, by Spectra-Physics). Its output is repetitionlowered by a pulse-picker and frequency-doubled in a BBO crystal to produce a 1.2-ps excitation source operating at a $4-\mathrm{MHz}$ repetition rate, and which can be tuned from 430 to $500 \mathrm{~nm}$. The laser beam enters the Nikon 2000 TE inverted microscope, being reflected toward the sample by the dichroic beamsplitter. The laser is focused on the back focal point of the objective and goes through the sample as a parallel beam, thus providing a wide-field excitation of the sample.
The fluorescence generated in the sample is guided to a time-resolved single-photon-counting photo-multiplier $\left(\mathrm{Q}_{\mathrm{A}}\right)$ from Europhoton Gmbh (Berlin http://www.europhoton.de/). During this process, half of the beam is cut by a mask. The other half reaches a $45^{\circ}$ removable Glan prism, which divides the fluorescence into the horizontal (transmitted) and vertical (reflected) polarization components. The vertical polarization is then flipped and redirected towards the detector by a pentaprism (Fig. 2). At the end of the line, the sensible surface of the detector receives a composite image comprising two spatially identical and symmetrical images of the sample that only differ in their polarization content.

$\mathrm{Q}_{\mathrm{A}}$ is a multi-channel plate photo-multiplier (PM) working in the single-photon counting mode. The anode is composed of five conductive parts. When a photoelectron is produced, an avalanche is created in the two multi-channel plates and spreads over the five anodes. The position of the photon on the photocathode can be calculated from a weighted mean of the five charges collected on the anodes. Based on this calculated coordinates, photons are sorted into $256 \times 256$ numerical pixels by rounding. This number of pixels fits with the observed precision in the determination of the position (100 $\mu \mathrm{m}$ for a $2.5-\mathrm{cm}$ large photocathode). For each photon detected, five values are measured and saved on a hard disk: the delay between the laser pulse and the arrival of the photon on the PM, the absolute arrival time (i.e. the time from the beginning of the measurement), the $X$ and $Y$ coordinates of the pixel representing the position of the photon on the photocathode and the total avalanche amplitude. The histogram of the number of photons collected per pixel gives the intensity image of the sample. The histogram of the number of photons collected as a function of absolute arrival time gives the evolution of the global fluorescence intensity and provides 


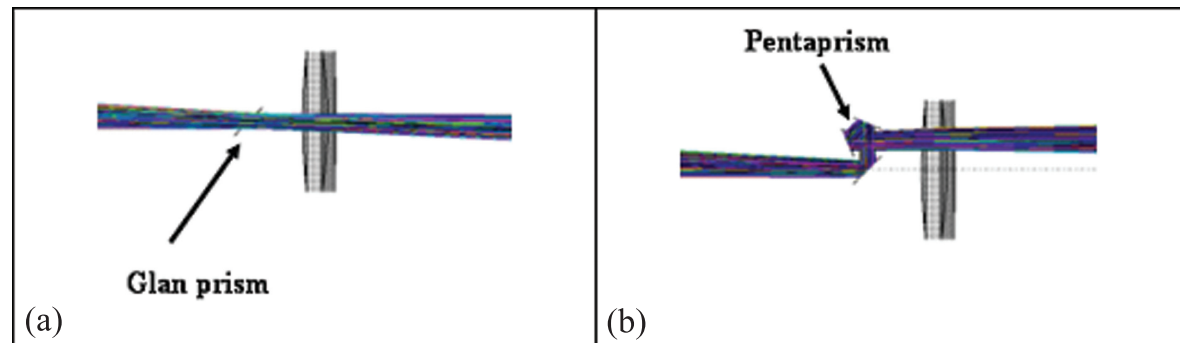

Fig. 2. Optical pathway in the polarization separator. (a) Beam pathway with a parallel polarization transmitted by the Glan prism and through the last lens. (b) Beam pathway with a perpendicular polarization reflected by the Glan prism, flipped and reoriented towards the detector by the pentaprism through the last lens.

a way to monitor photobleaching if the excitation intensity remains constant during the experiment. The histogram of the total number of photons as a function of the delay between the laser pulse and the fluorescence gives the global fluorescence decay of the sample. Some kinds of histograms can also be constructed for selected areas of the image, thus giving the specific fluorescence decay of the selected area. For each pixel we also calculate the sum of all the delays between the laser pulse and the collected photons. When divided by the number of photons arrived on each pixel this sum of all the delays gives the average fluorescence lifetime as a FLIM image. This is a fast and robust way to make FLIM images.

The time-resolved anisotropy function, $r(t)$, is then calculated (without deconvolution of the instrument response function) from the Perrin equation:

$$
r(t)=\frac{I_{\|}-G I_{\perp}}{I_{\|}+2 G I_{\perp}}
$$

where $G$ is the apparatus function and $I_{\perp}$ and $I_{\|}$are the perpendicular and parallel components of the fluorescence polarization. $G$ represents the ratio of the sensitivity of the detection line for the two polarizations. In our case, it will change from pixel to pixel. It is determined on a reference sample emitting equal intensity for both polarizations (long life-time fluorophore such as rhodamine in a fluid sample such as water).

\section{Results and discussion}

We present here the different aspects of the set-up characterization. First, we shall focus on the fluorescence lifetime and intensity measurements. Then, we will characterize the space resolution of this new detector. Finally we will discuss the measurement and imaging of fluorescence anisotropy.

\section{Time measurement}

Lifetime measurement correction. The crude FLIM image of a homogeneous sample [Rhodamine 6G (Magde et al., 2002), $10^{-4} \mathrm{Min}$ water] is shown in Fig. 3a. It shows a lifetime between 3 ns (black pixel) and $3.6 \mathrm{~ns}$ (white pixel), with more white pixels in the middle of the image. This homogeneous sample should show a sharp distribution in fluorescencelifetime. Thus, when the centre of the image seems to have a longer lifetime, the periphery shows a shorter one. The artificial delays that are observed are due to electronics. Indeed for each avalanche,

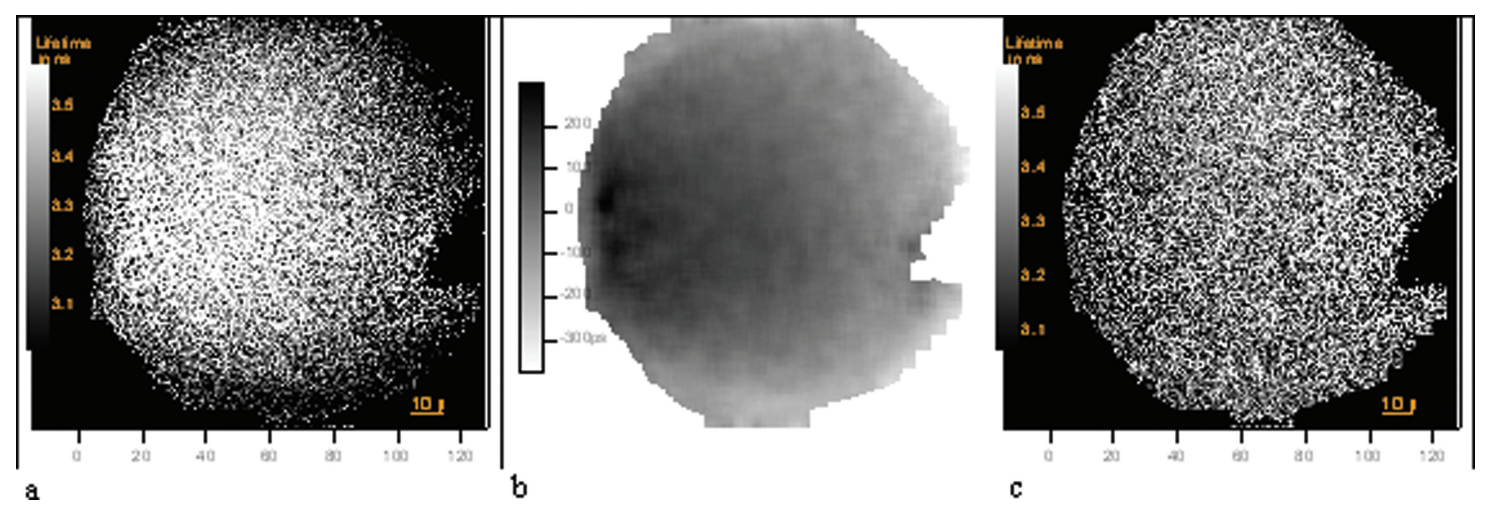

Fig. 3. Time transit correction. (a) Crude image of rhodamine $6 \mathrm{G}$ in water sample. (b) Time transit correction map obtained by the difference between the time measured on each pixel from the "crude image" and the average value, which is the expected lifetime. (c) Corrected image of the first sample using the time transit correction map. 


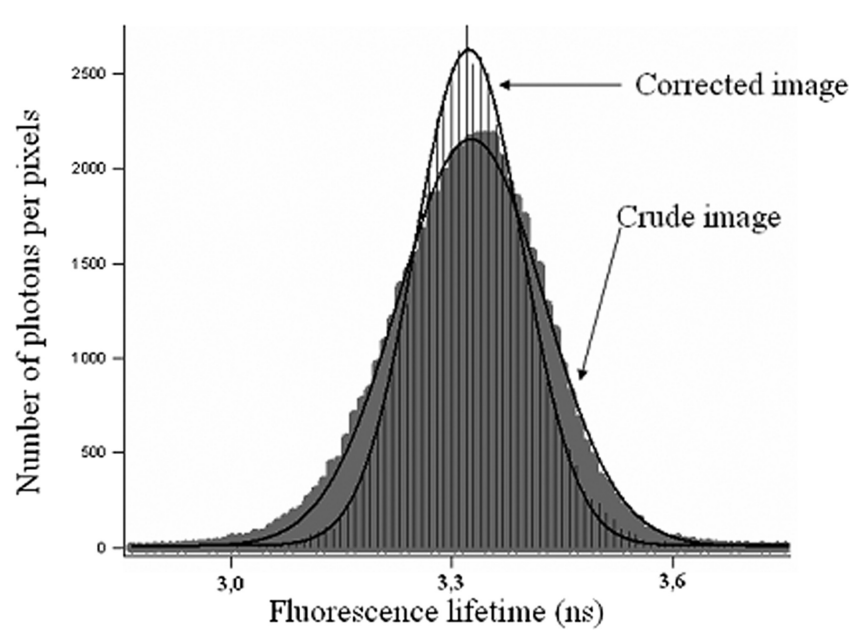

Fig. 4. Histogram of the collected photons vs. lifetime before and after correction of time transit. The sample is rhodamine $6 \mathrm{G}$ in water.

the propagation time of the induced current pulse through the multi-channel plate to reach the discriminator depends on the location of the avalanche. To correct those constant time-transit variations, we use a time-transit correction map, which has been stored and is then processed for any further experiments. To build up this map, we have used a rhodamine sample in water that has a homogeneous lifetime on the whole sample and can be thus used as a reference. We calculate the difference between the time measured on each pixel from the "crude image" referred above and the average value, which is the expected lifetime; this difference will then be automatically subtracted from all future FLIM images.

Dispersion of the average fluorescence time. If we look at Fig. 3c, we see a uniform value for the measure of fluorescence lifetime, but a remaining distribution (Fig. 4). We shall show that this residual dispersion is due to photon statistics.

The precision of average fluorescence time measurements depends upon the number of photons collected by each pixel of the image. In the case of a monoexponential decay (e.g. rhodamine), the distribution $P(t)$ of the photon arrival time is $k$.exp $(-k t)$ were $1 / k$ is the fluorescence lifetime. The expected value of the mean arrival time of $N$ photons is $1 / k$ and the variance of these arrivals times is $1 / k^{2}$. The variance of the mean arrival time of $N$ photons will be $1 /(k N)$. This variance will tend to zero as $N$ approaches infinity. Thus, the number of photons per pixel has been counted and an average fluorescence time distribution has been determined according to this number of photon per pixels. We get a family of Gaussians whose widths give the lifetime dispersion as a function of the number of photons (Fig. 5). The width scales linearly with $1 / \sqrt{N}$ and extrapolates to $39 \mathrm{ps}$. This shows that the dispersion of fluorescence time is due to photon statistics and the extrapolated value to infinite photon count gives the quality of the correction by the transit time map.

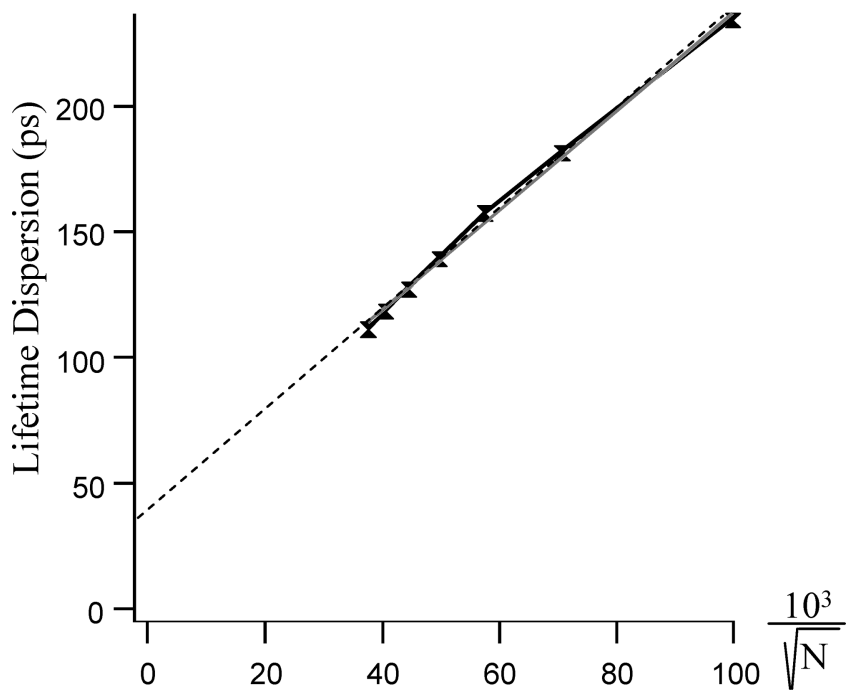

Fig. 5. Evolution of the lifetime dispersion with the number of photons per pixel. The pixels of an image of a rhodamine sample were sorted according to their intensity and the lifetime distribution was fitted with a Gaussian whose width is the lifetime dispersion.

\section{Temporal resolution}

The temporal resolution of the set-up is limited by our detector. To determine it, we use a 1.2-ps pulse onto a microscope slide and measure the time response of our detector (Fig. 6). The middle height width of the recorded pulse is around 310 ps. This is the shortest event we can efficiently measure and it

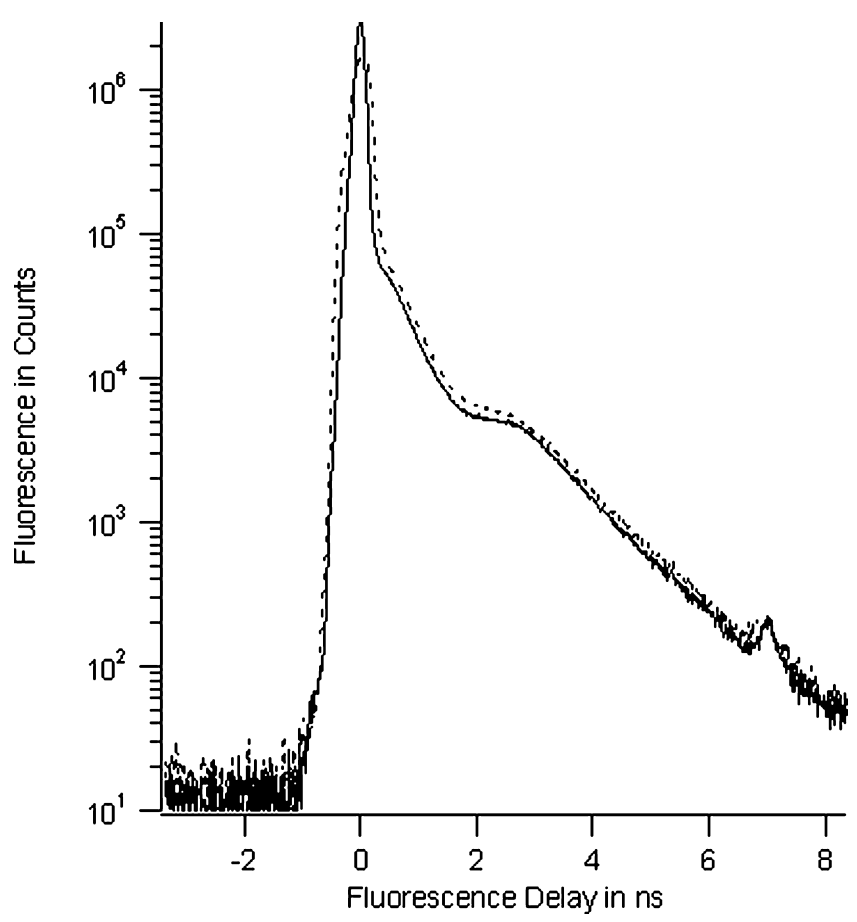

Fig. 6. Pulse (dashed line) and corrected pulse (plain line). Laser is directed onto a microscope slide. 


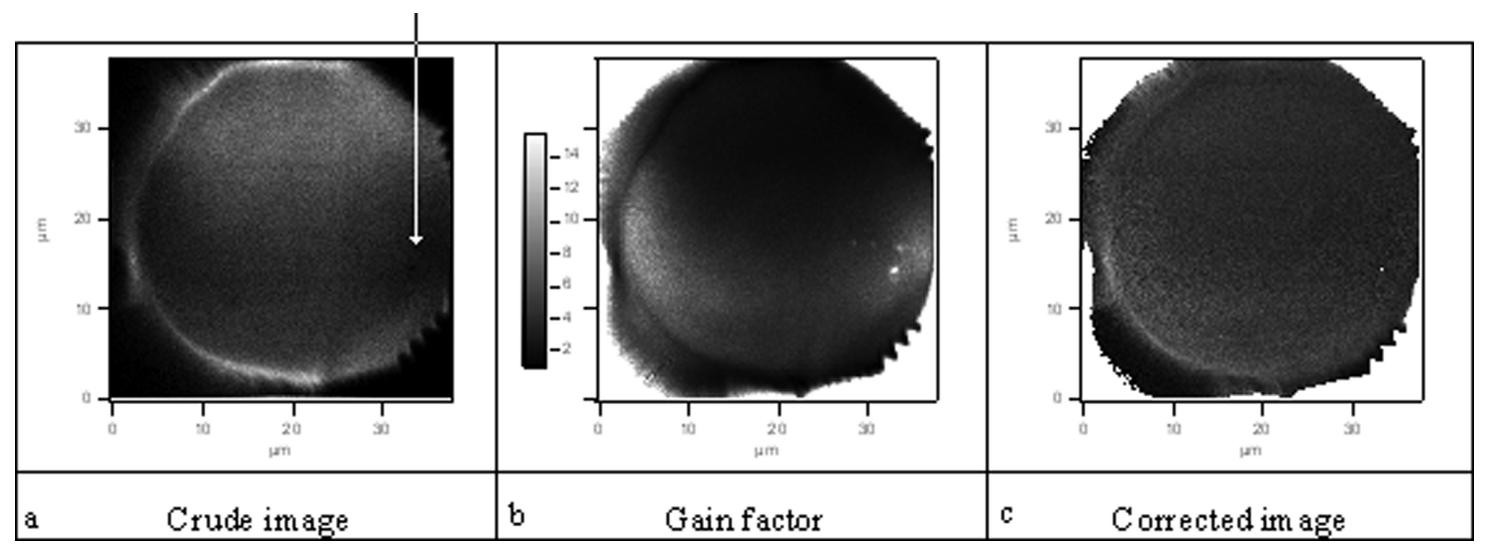

Fig. 7. Gain correction. (a) Crude image of a rhodamine in water sample. (b) Gain factor correction map calculated from another sample. (c) Corrected image of the first sample using the gain factor correction map. The arrow indicates the small dark spots.

gives us an idea of our temporal resolution. Global fluorescence decays can also be corrected from the transit time deviation. After this correction, the full width at half maximum of the pulse reaches the value of $150 \mathrm{ps}$. It can be reduced to $130 \mathrm{ps}$ only if the centre of the detector is used. This time resolution agrees with the one expected from the diameter of the holes in the MCPs. ${ }^{1}$

At this point, we can note also in Fig. 6 the large dynamic range over 5 orders of magnitude, which is an excellent feature related to the very low dark count rate of the detector (15-20 cps).

Time shift from the microscope objectives. The use of four different objectives leads to another small time shift from one objective to another because, though objectives are designed to perform with the same work distance, the lenses sizes are different. This explains why the way through the $\times 40$ is shorter than the other $(-75 \mathrm{ps})$ whereas the $\times 100$ induces an extra time of 65 ps. Again, those shifts are automatically corrected to set all pulses at the same point.

\section{Intensity measurements}

Gain correction. Some areas of our detector can present different gains. The lighting of the sample may also affect the intensity image because some areas might be more lighted than others. As previously mentioned, a software correction is necessary to supplement these sensitivity variations (Fig. 7). The gain factor map is calculated from a rhodamine in aqueous sample.

N.B. 1: On the right part of the uncorrected image (Fig. 7a), we can notice small dark spots that are areas of the detector that are less sensible than the average.

\footnotetext{
1 Personal communication and unpublished data from James Milnes (Photek). See also PMT section in http://www.photek.com/
}

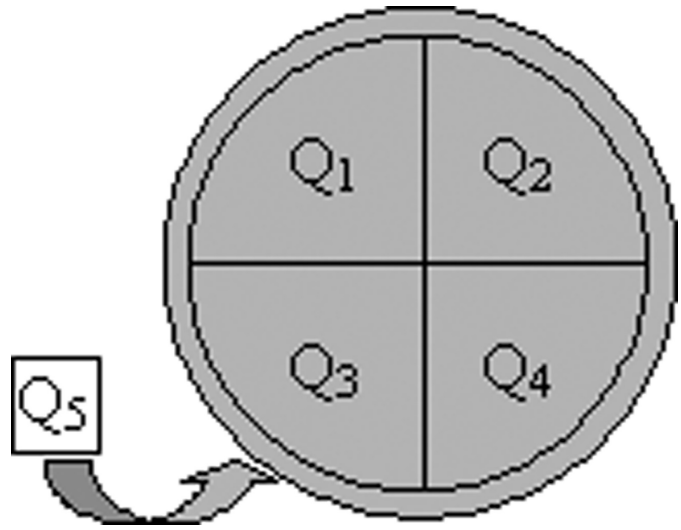

Fig. 8. Position of the anodes in the detector.

N.B. 2: The overload on the periphery of the image is neither due to reflections on the inner walls of our set-up nor due to accumulation of miscalculated photons, thanks to the fifth surrounding anode (Fig. 8).

Dead time. Once a photon reaches the anode, it has to be proceeded by the set-up. During this dead-time, any other photon will not be taken into account. This dead-time is determined by sorting the acquired photons according to their delay relative to the previous photons (Fig. 9). In Fig. 9, the insert is a magnification of the five first microseconds in which we can measure a dead time of $2.15 \mu \mathrm{s}$.

Saturation. The histogram (data not shown) of the amplitude of avalanches is independent of count rate. Thus the saturation of the count rate is not due to a saturation of the MPC channel. The saturation due to the $2.15 \mu$ s dead time of the charge amplifiers should be $465 \mathrm{kHz}$. A lower saturation rate is observed and can be evaluated to $72 \mathrm{kHz}$ by a simple monoexponential fit (Fig. 10). This saturation might be due to the data transfer later in the process. 


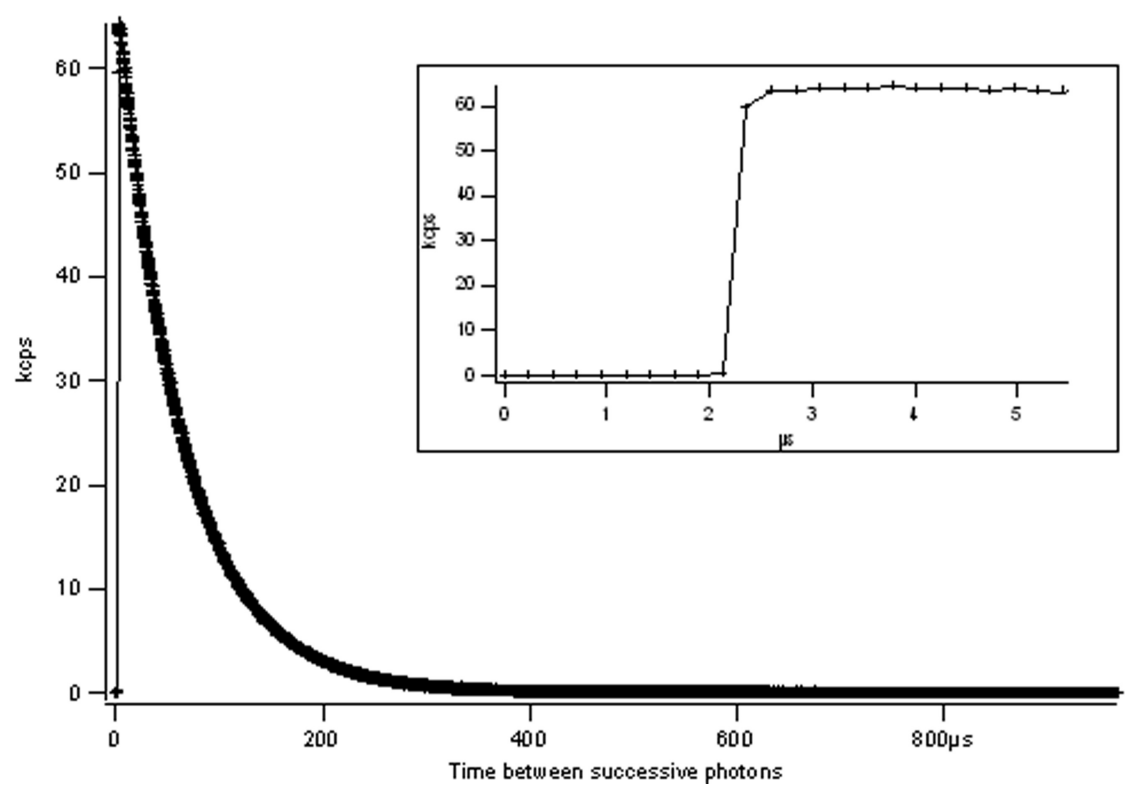

Fig. 9. Determination of the dead time. The acquired photons are sorted according to their delay relative to the previous photon. The insert is a magnification of the five first microseconds.

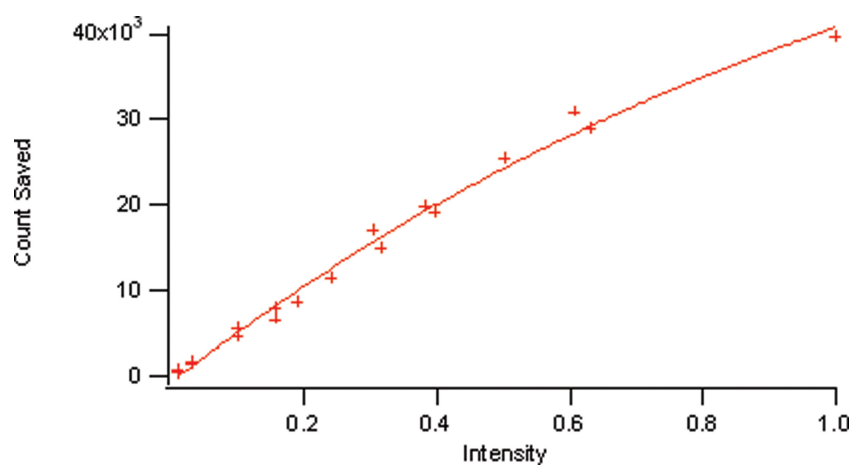

Fig. 10. Saturation curve. The line is the monoexponential fit of the saturation curve.

\section{Spatial resolution}

The determination of the spatial resolution of the device was carried out by imaging isolated fluorescent nanolatex. These latexes, from Molecular Probes (F-8787), have a diameter of $20 \mathrm{~nm}$, which is below the resolution. The size of the smallest spots gives us the spatial resolution. The latexes are spin-coated on a microscope slide after dissolution ( $0.2 \%$ in mass) in a $1 \%$ polyvinyl alcohol aqueous solution according to the protocol described by Méallet-Renault et al. (2000): (1) $5 \mathrm{~s}$ at $300 \mathrm{rpm}$ and (2) $1 \mathrm{~min}$ at $1600 \mathrm{rpm}$ ). Thanks to a piezoelectric plate, a series of images are recorded at different heights above and under the focus plan (Fig. 11).

The smallest fluorescent marks, corresponding to isolated nanolatex particles, are fitted by a series of two-dimensional
Gaussians whose widths are plotted as a function of the altitude in Fig. 12. The lowest point of the resultant curve corresponds to the focus plan and the size of the spot $(200 \mathrm{~nm})$ is the spatial resolution of the imager. The Gaussian half-width of $200 \mathrm{~nm}$ corresponds to an Airy radius of $400 \mathrm{~nm}$. The pixel size is smaller than the resolution limit and does not limit the overall spatial resolution of the set-up.

NB: The dissymmetry of the curve Fig. $12 b$ is due to the imperfectly corrected spherical aberration of our set-up.

\section{Anisotropy}

Delay due to the set-up. The two different polarizations of the emitted fluorescence have different courses through the setup. Indeed, the vertical component is reflected by the Glan prism and then by the pentaprism. Consequently, the optical pathway of this polarization is longer than the other, leading to an extra delay of $160 \mathrm{ps}(4.8 \mathrm{~cm}$ Fig. 13). Before making any calculations on these decays, such as for anisotropy, this shift is corrected by computer post-treatment. A typical corrected FLIM image of the two polarizations of rubrene fluorescence is presented in Fig. 14. No deconvolution is necessary because fluorophores used in this study have long fluorescent lifetimes.

Effect of the collection angles. Since we intend to use different scales of sample, we use different microscope objectives $(\times 10$, $\times 20, \times 40, \times 60$ and $\times 100)$. Those objectives have different 


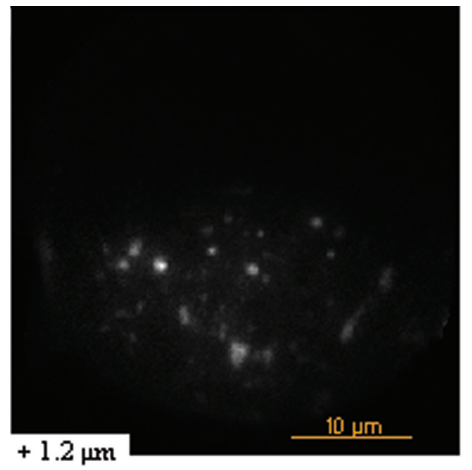

a

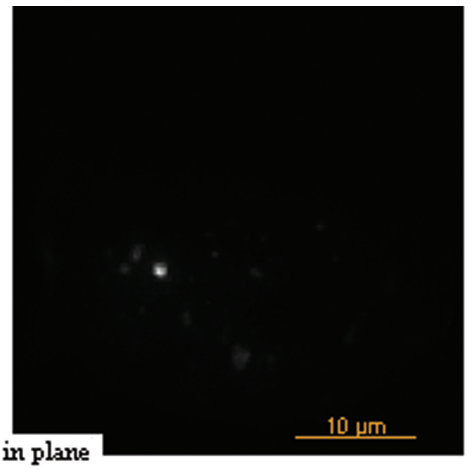

$\mathrm{b}$

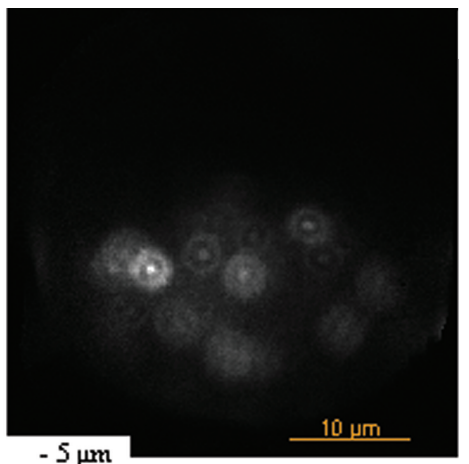

C

Fig. 11. Images of spin-coated 20-nm fluorescent nanolatex in PVA film. (b) is the image in focal plane, (a) is the image $1.2 \mu \mathrm{m}$ underneath and (c) is the image $5 \mu \mathrm{m}$ over the focal plane.

numerical aperture (resp. 0.3, 0.45, 0.6, 0.95 and 1.45). It is well known that measured anisotropies depend on the collection angle. For high collection angles, we start to collect the light emitted by the $x$-oriented population. Thus the observed anisotropy is reduced. This collection angle $(u)$ is a function of the numerical aperture of the objective and of the refractive index in the fluorescent solution (n').

$$
\sin u=\frac{\mathrm{NA}}{n^{\prime}} .
$$

We have studied the effect of the depolarization of the anisotropy of a solution of rubrene in squalane [n' $=14474$ (Tripathi, 2005)] with these increasing collection angles. (Figs. 15 and 16, and Table 1). The anisotropy measured in a cuvette within a calibrated spectrofluorometer has been added with a numerical aperture of 0 . The predicted anisotropy value has been calculated by a full-vectorial modelling, which is based on a method proposed by Enderlein (2000). The method permits the calculation of the electric field (Ei) (in the image space) emitted by a fluorophore (i) in the object space. It depends on the position and the orientation of the emitting dipole. The index of the fluorescent solution, the polarization of the excitation beam and the characteristics of the microscopes (NA, magnification) and of the fluorophores are taken into account too. In this case, the emitted field is projected along the two perpendicular directions of polarization (one is parallel to the polarization excitation beam). Therefore in the focal plane of detection, we can calculate two intensity values $\left(I i_{\text {II }}=\left\|\mathbf{E i}_{\text {II }}\right\|^{2}\right.$ and $I i_{\perp}=\left\|\mathbf{E i}_{\perp}\right\|^{2}$ ) corresponding to the two analysis directions of polarization. Fluorescent objects are spatially incoherent; thus the intensity on the photo-detector is equal to the sum of all intensities emitted by each fluorophore of the solution. In this case and to take into account the random orientation of fluorophores, we have integrated the two intensity values (Ii and $\mathrm{Ii}_{\perp}$ ) on all possible orientations (three angles) and on the volume (three directions) of the object. One can note that three

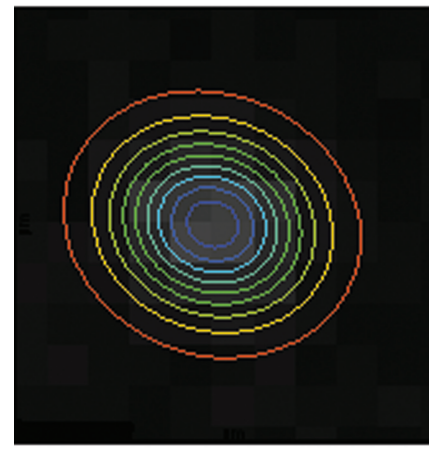

(a)

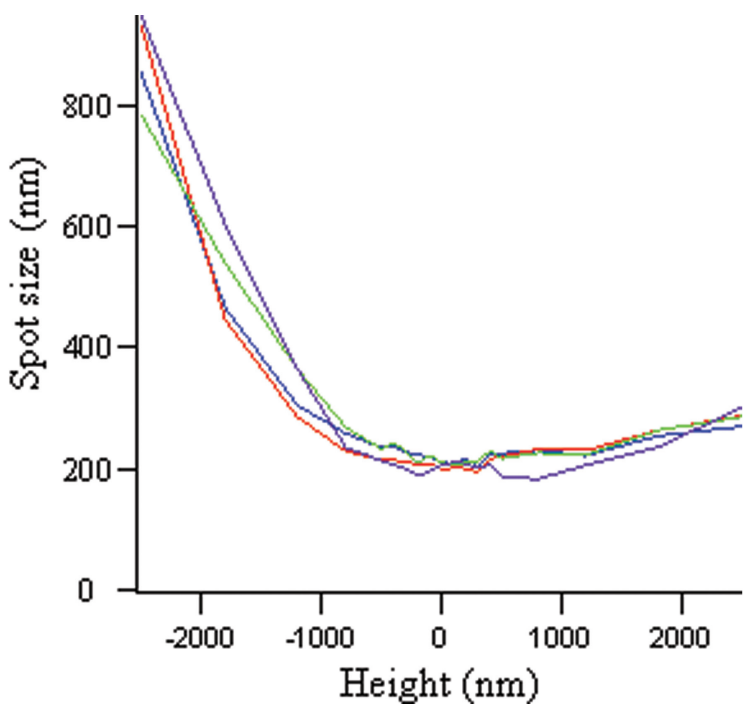

(b)

Fig. 12. (a) Two-dimensional Gaussian fit of the fluorescent spot of a 20-nm fluorescent nanolatex. (b) Gaussian's width vs. altitude. 


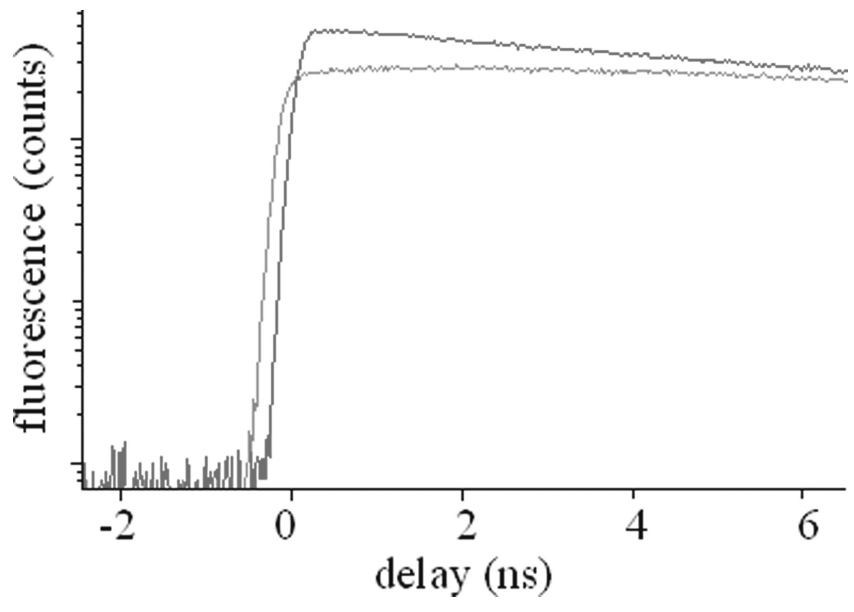

Fig. 13. Parallel and perpendicular decays of rubrene fluorescence. Fluorescence decays of rubrene in solution in squalane, before correction of the pathway shift.

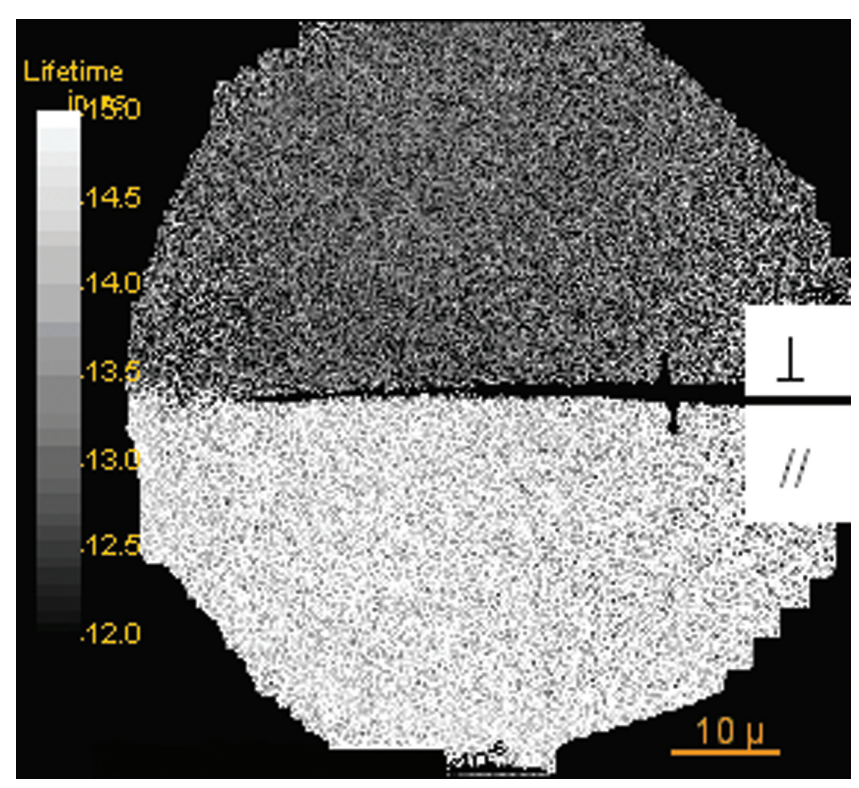

Fig. 14. FLIM image of the two polarization components of the fluorescence of rubrene in squalane. In this composite image, the perpendicular component of the fluorescence is at the top and the parallel component is at the bottom. The apparent difference in average lifetime is the signature of the fluorescence anisotropy.

angles are necessary to describe completely the orientation of the fluorophore with its absorption dipole, which is not collinear to the emission dipole. Therefore we can calculate the anisotropy from the two intensity values $\left(I_{\|}\right.$and $\left.I_{\perp}\right)$ derived from the two triple integrals for different fluorophores and different microscope objectives. The predicted decrease of anisotropy (Axelrod, 1979) is observed as previously stated with a different configuration (Schoutteten et al., 1997) but does not prevent anisotropy measurement up to a collection angle of $50^{\circ}$, where it can be neglected as reported by Seigel et al. (2003).

The time evolution of polarized fluorescence intensity is of two types, $I_{\|}$and $I_{\perp}$ (Fleming, 1986):

$$
\begin{gathered}
I_{\|}=I_{0} \exp \left(-\frac{t}{\tau_{\text {rot }}}\right)(1+2 r(t)) \\
I_{\perp}=I_{0} \exp \left(-\frac{t}{\tau_{\text {rot }}}\right)(1-r(t))
\end{gathered}
$$

When high collection angles are used, the collected intensity with a vertical (resp. horizontal) analyzer is a linear combination of $I_{\|}$and $I_{\perp}$. Thus, the characteristic rotational correlation times are not affected by the collection angle used.

Anisotropy in thin films. Measurements of anisotropy in films will depend also on the collection angle but with different relations since the radiation pattern is no longer toroidal. Spincoated 50-nm films of a fluorescent dye (PM567 $30 \mathrm{\mu g} \mathrm{g}^{-1}$ in $10 \%$ PMMA in toluene; (1) $10 \mathrm{~s}$ at $1000 \mathrm{rpm}$ and (2) $50 \mathrm{~s}$ at $3000 \mathrm{rpm}$ ) have been studied with the different objectives (Fig. 17). The relaxation of the anisotropy observed in such a film is not due to the Brownian motion of the excited state in the polymer film but to the Förster resonance energy transfer (FRET) from the excited molecule to one of its neighbour. The angular distribution of the transition moment after one FRET jump can be calculated. For a given distance $R$, the jump probability is proportional to

$$
\kappa^{2}=\left[3\left(\vec{u} \cdot \vec{p}_{0}\right)\left(\vec{u} \cdot \vec{p}_{1}\right)-\left(\vec{p}_{0} \cdot \vec{p}_{1}\right)\right]^{2} .
$$

The angular distribution $\rho(\theta, \phi)$ of the transition dipole after one jump is given by averaging $\kappa^{2}$ over $\vec{u}$. This average gives a distribution $\rho(\theta, \phi)=\left(3+\cos ^{2}(\theta)\right)$, which corresponds to a reduction of anisotropy down to a value of $4 \%$ of the original value (Agranovich \& Galanin, 1982). Some authors show that this is zero (Berberan-Santos \& Valeur, 1991). Thus the rate of the anisotropy relaxation is the rate of the energy transfer to the first neighbour (Förster, 1959; Millar et al., 1981):

$$
r(t)=r_{0} \exp \left(-\sqrt{\frac{t}{\tau}}\right) .
$$

Anisotropy decays have been fitted to this equation leading to the initial anisotropy $\left(r_{0}\right)$ and the characteristic time of the energy transfer $(\tau)$ gathered in Table 2. Apart from the highest numerical aperture, the anisotropy depolarization can also be neglected.

\section{Conclusion}

We describe a robust and useful set-up to measure fluorescence anisotropy imaging. The whole decay profile of the two polarizations is acquired simultaneously with a single-photon counting system. Thus, there is no sampling of the fluorescence 


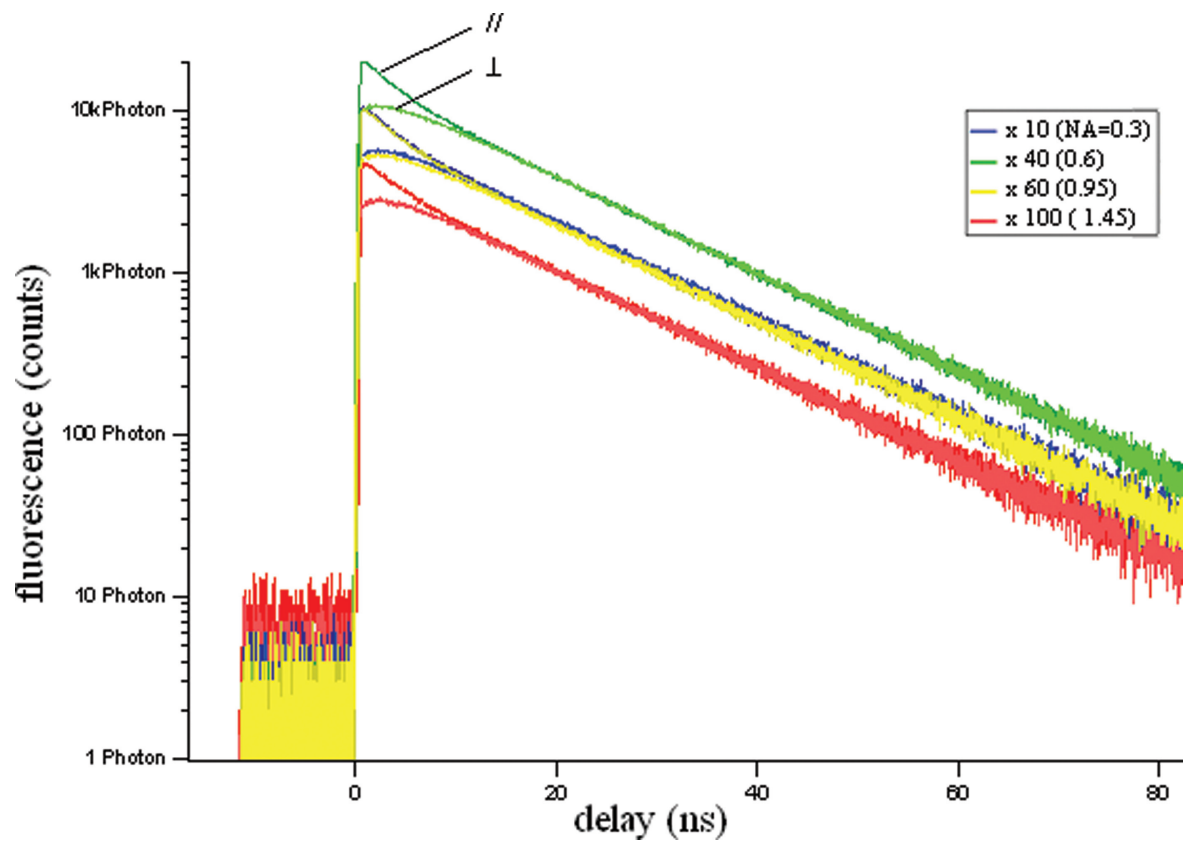

Fig. 15. Fluorescence decays of rubrene in squalane. The decays have been recorded using four different microscope objectives and numerical apertures. The decay for parallel polarization (resp. perpendicular) has been calculated from the upper part (resp. bottom part) of the image.

and the two polarizations are acquired at the same time, with the same sample, at the same position, with the same experimental conditions preventing us from artefacts such as instrument drift, evolution of the sample and photobleaching. Since FLIM can be estimated with a limited number of photons,
FLIM images can be recorded with a single-photon counting system in less than 1 minute. Correction maps are required for gain and transit time. These correction maps appear to be stable for a few months and recalibration of the set-up is easy on a few reference samples. Overall, this device appears to be a powerful

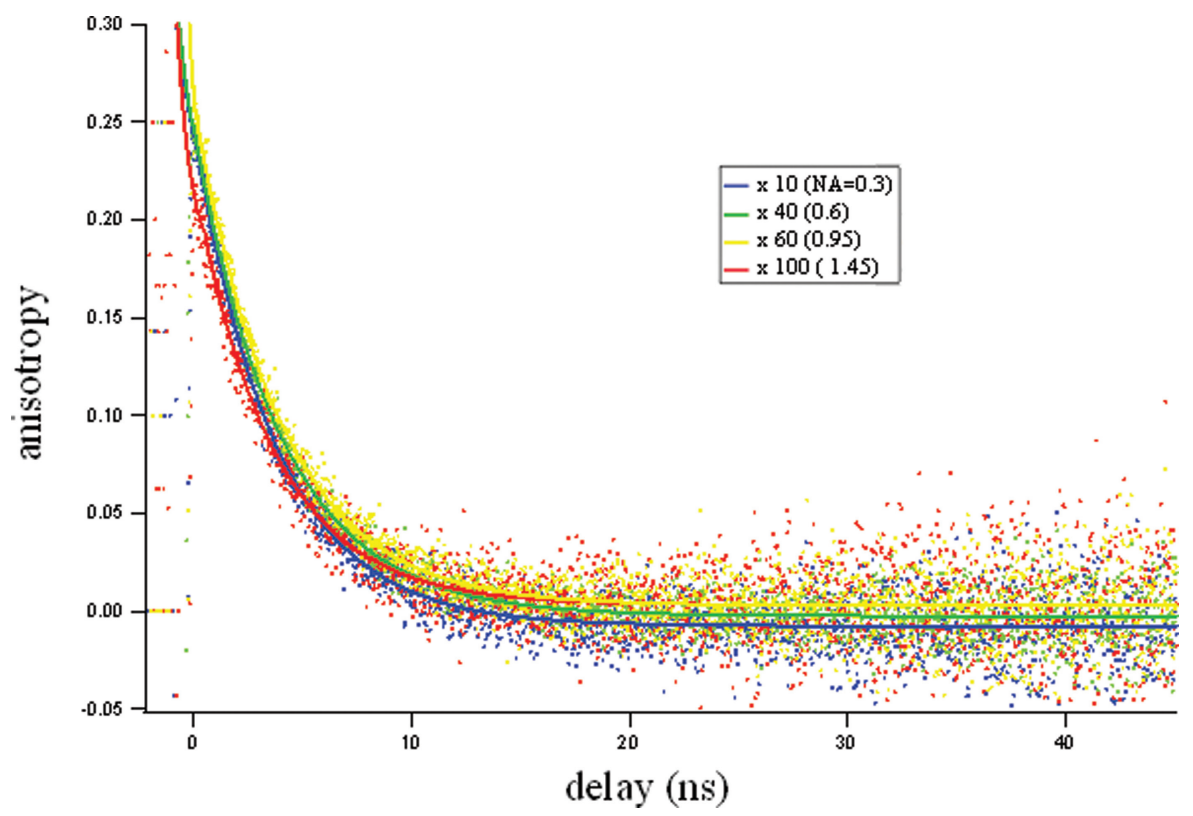

Fig. 16. Time-resolved anisotropy of rubrene in squalane with different numerical apertures. The anisotropy has been calculated according to the Eq. 1 from the decays recorded using 4 different microscope objectives. 


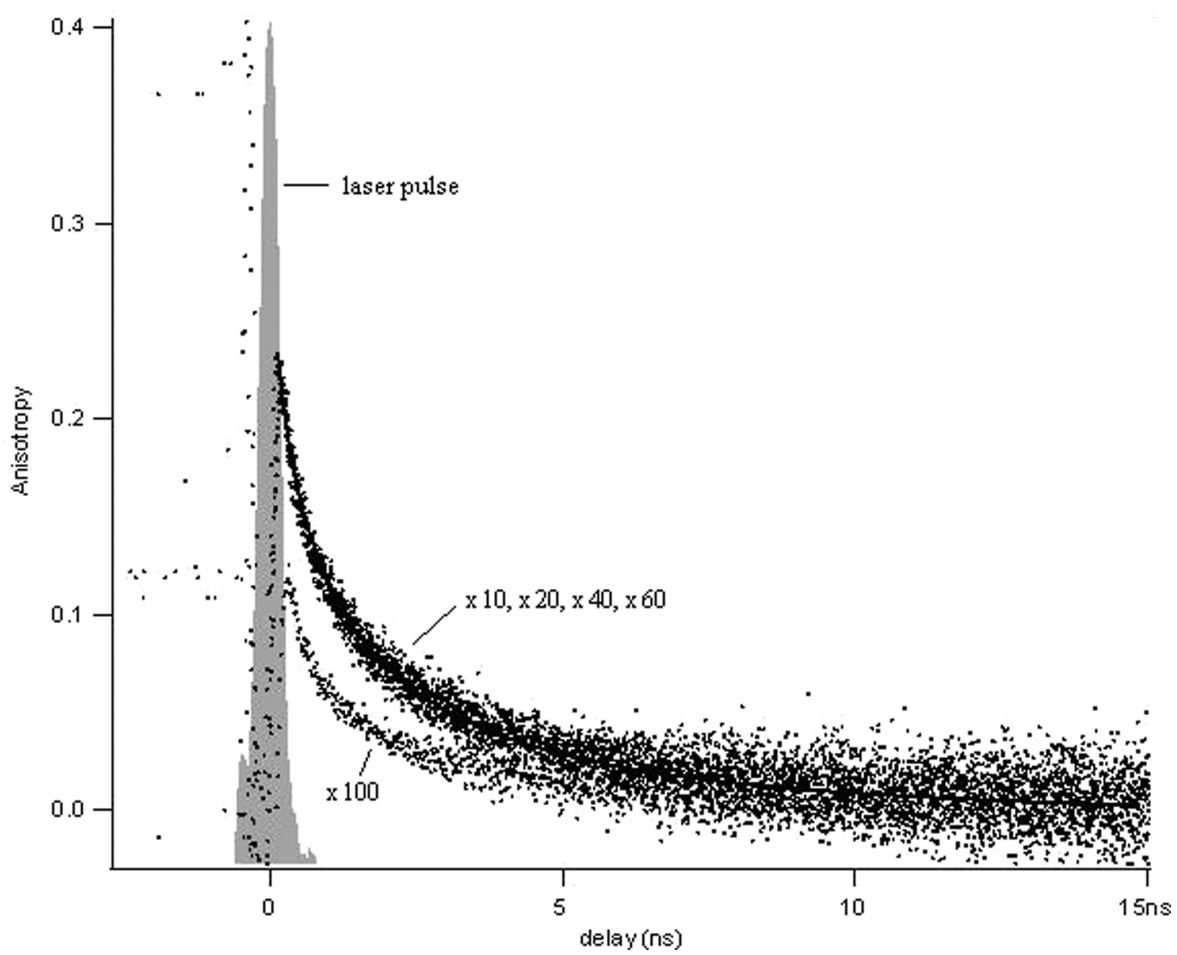

Fig. 17. Time-resolved anisotropy of PM567 in PMMA film with different numerical aperture. The anisotropy has been calculated according to Eq. (1) from the decays recorded using five different microscope objectives. The laser pulse has been superimposed to the figure.

Table 1. Time zero anisotropy $\left(r_{0}\right)$ of rubrene in squalane with different numerical apertures (NA); calculated anisotropy $\left(r_{\mathrm{C}}\right)$; rotational correlation time $\left(\tau_{\text {rot }}\right)$

\begin{tabular}{llcll}
\hline Objective & NA & $r_{0}$ & \multicolumn{1}{c}{$r_{\mathrm{c}}$} & $\tau_{\text {rot }}(\mathrm{ns})$ \\
\hline 0 & 0 & 0.297 & - & 2.65 \\
10 & 0.3 & 0.281 & 0.318 & 2.66 \\
40 & 0.6 & 0.272 & 0.311 & 2.52 \\
60 & 0.95 & 0.283 & 0.293 & 2.50 \\
100 & 1.45 & 0.234 & 0.244 & 2.66 \\
\hline
\end{tabular}

and sensible tool to do imaging in different fields such as cellular imaging, chemical sensing, $\mu \mathrm{TAS}$, biochip diagnostics. It could be specifically interesting for applications in which optical sectioning and scanning are inconvenient, such as the evaluation of the polarization properties of evanescent waves in TIRF-trFAIM (Gee et al., 2004).

\section{References}

Agranovich, V.M. \& Galanin, M.D. (1982) Electronic Excitation Energy Transfer in Condensed Matter. Elsevier, Amsterdam.

Axelrod, D. (1979) Carbocyanine dye orientation in red cell membrane studied by microscopic fluorescence polarization. Biophys. J. 26(3), $557-$ 573 .
Table 2. Time zero anisotropy $\left(r_{0}\right)$ of PM 567 in PMMA films with different numerical apertures (NA) and characteristic time of the energy transfer $(\tau)$

\begin{tabular}{llccc}
\hline Objective & NA & $r_{0}$ & $r_{\mathrm{C}}$ & $\tau(\mathrm{ps})$ \\
\hline 10 & 0.3 & 0.348 & 0.330 & 865 \\
20 & 0.45 & 0.335 & 0.327 & 865 \\
40 & 0.6 & 0.332 & 0.322 & 819 \\
60 & 0.95 & 0.344 & 0.304 & 807 \\
100 & 1.45 & 0.213 & 0.252 & 739 \\
\hline
\end{tabular}

Berberan-Santos, M.N. \& Valeur, B. (1991) Fluorescence depolarization by electronic energy transfer in donor-acceptor pairs of like and unlike chromophores. J. Chem. Phys. 11, 8048-8055.

Buurman, E.P., Sanders, R., Draaijer, A., Gerritsen, H.C., van Veen, J.J.F., Houpt, P.M. \& Levine, Y.K. (1992) Fluorescence lifetime imaging using a confocal laser scanning microscope. Scanning 14, 155159.

Clayton, A.H., Hanley, Q.S., Arndt-Jovin, D.J., Subramaniam, V. \& Jovin, T.M. (2002) Dynamic fluorescence anisotropy imaging microscopy in the frequency domain (rFLIM). Biophys. J. 83(3), 1631-1649.

Emiliani, V., Sanvitto, D., Tramier, M., et al. (2003) Low-intensity twodimensional imaging of fluorescence lifetimes in living cells. Appl. Phys. Lett. 83(12), 2471-2473.

Enderlein, J. (2000) Theoretical study of detecting a dipole emitter through an objective with high numerical aperture. Opt. Lett. 25, 634-636. 
Fleming, G.R. (1986) Chemical Application of Ultrafast Spectroscopy, Oxford University press, Oxford.

Förster, T. (1959) Transfer mechanisms of electronic excitation. Disc. Faraday Soc. 27, 7-17.

Gadella, T.W.J., Jovin, T.M. \& Clegg, R.M. (1993) Fluorescence lifetime imaging microscopy (FLIM): spatial resolution of microstructures on the nanosecond time scale. Biophys. Chem. 48(1), 221-239.

Gautier, I., Tramier, M., Durieux, C., et al. (2001) Homo-FRET microscopy in living cells to measure monomer-dimer transition of GFP-tagged proteins. Biophys. J. 80(6), 3000-3008.

Gee, M.L., Lensun, L., Smith, T.A. \& Scholes, C.A. (2004) Time-resolved evanescent wave-induced fluorescence anisotropy for the determination of molecular conformational changes of proteins at an interface. Eur. Biophys. J. 33(2), 130-139.

Ha, T., Laurence, T.A., Chemla, D.S. \& Weiss, S. (1999) Polarization spectroscopy of single fluorescent molecules. J. Phys. Chem. B 103(33), 6839-6850.

Herman, B., Wang, X.F., Wodnicki, P., Perisamy, A., Mahajan, N., Berry, G. \& Gordon, G. (1999) Fluorescence lifetime imaging microscopy. Applied Fluorescence in Chemistry, Biology and Medicine (eds. by W. Rettig, B. Strehmel, S. Schrader \& H. Seifert) pp. 491-507. Springer, Berlin.

Lakowicz, J.R. (1999) Principles of Fluorescence Spectroscopy. Kluwer Academic/Plenum Publishers, New York.

Magde, D., Wong, R. \& Seybold, P.G. (2002) Fluorescence quantum yields and their relation to lifetimes of rhodamine $6 \mathrm{G}$ and fluorescein in nine solvents: improved absolute standards for quantum yields. Photochem. Photobiol. 75(4), 327-334.

Méallet-Renault, R., Yoshikawa, H., Tamaki, Y., Asahi, T., Pansu, R.B. \& Masuhara, H. (2000) Confocal microscopic study on fluorescence quenching of single latex beads in poly(vinyl alcohol) film. Polym. Adv. Technol. 11(8-12), 772-777.

Millar, D.P. (1996) Time-resolved fluorescence spectroscopy. Curr. Opin. Struct. Biol. 6(5), 637-642.

Millar, D.P., Robbins, R.J. \& Zewail, A.H. (1981) Picosecond dynamics of electronic energy transfer in condensed phases. J. Chem. Phys. 75(8), 3649-3659.
Miyawaki, A., Llopis, J., Heim, R., McCaffery, J.M., Adams, J.A., Ikura, M. \& Tsien, R.Y. (1997) Fluorescent indicators for $\mathrm{Ca}^{2+}$ based on green fluorescent proteins and calmodulin. Nature 388(6645), 882887.

Schaffer, J., Volkmer, A., Eggeling, C., Subramaniam, V., Striker, G. \& Seidel, C.A.M. (1999) Identification of single molecules in aqueous solution by time-resolved fluorescence anisotropy. J. Phys. Chem. A 103(3), 331336.

Schoutteten, L., Denjean, P., Joliff-Botrel, G., Bernard, C., Pansu, D. \& Pansu, R.B. (1999) Development of intracellular calcium measurement by time-resolved photon-counting fluorescence. Photochem. Photobiol. 70(5), 701-709.

Schoutteten, L., Denjean, P. \& Pansu, R.B. (1997) Characterisation of a time resolved photon counting confocal fluorescence microscope. J. Fluoresc. 7(2), 155-165.

Siegel, J., Suhling, K., Leveque-Fort, S., et al. (2003) Wide-field timeresolved fluorescence anisotropy imaging (TR-FAIM): imaging the rotational mobility of a fluorophore. Rev. Sci. Instrum. 74(1), 182192.

So, P.T., French, T., Yu, W., Berland, K., Dong, C.Y. \& Gratton, E. (1995) Time-resolved fluorescence microscopy using two-photon excitation. Bioimaging 3, 49-63.

So, P.T., Konig, K., Berland, K., et al. (1998) New time-resolved techniques in two-photon microscopy. Cell. Mol. Biol. (Noisy-le-grand) 44(5), 771793.

Tramier, M., Kemnitz, K., Durieux, C., Coppey, J., Denjean, P., Pansu, R.B. \& Coppey-Moisan, M. (2000) Restrained torsional dynamics of nuclear DNA in living proliferative mammalian cells. Biophys. J. 78(5), 26142627.

Tripathi, N. (2005) Densities, viscosities, and refractive indices of mixtures of hexane with cyclohexane, decane, hexadecane, and squalane at 298.15K. Int. J. Thermophys. 26(3), 693-703.

Valeur, B. (2001) Molecular Fluorescence: Principle and Applications. Wiley, New York.

Wang, X.F., Perisamy, A. \& Herman, B. (1992) Fluorescence lifetime imaging microscopy (FLIM): instrumentation and applications. Crit. Rev. Anal. Chem. 23, 369-395. 